\title{
Inkontinenz beim Hund chiropraktisch behandeln - 3 Fallberichte
}

Ute Körner

Der Leidensdruck bei Harninkontinenz ist auf beiden Seiten hoch - sowohl natürlich auf der Seite des Tieres als auch auf der des Besitzers. Die Behandlung ist nicht immer einfach, doch es gibt Möglichkeiten, zumindest Linderung zu verschaffen. Dafür sollte man die Hintergründe kennen und verstehen und wissen, wie und wo man ganzheitlich manualtherapeutisch ansetzen kann. Bei inkontinenten Patienten ist außerdem die richtige Kommunikation mit dem Patientenbesitzer von großer Bedeutung.

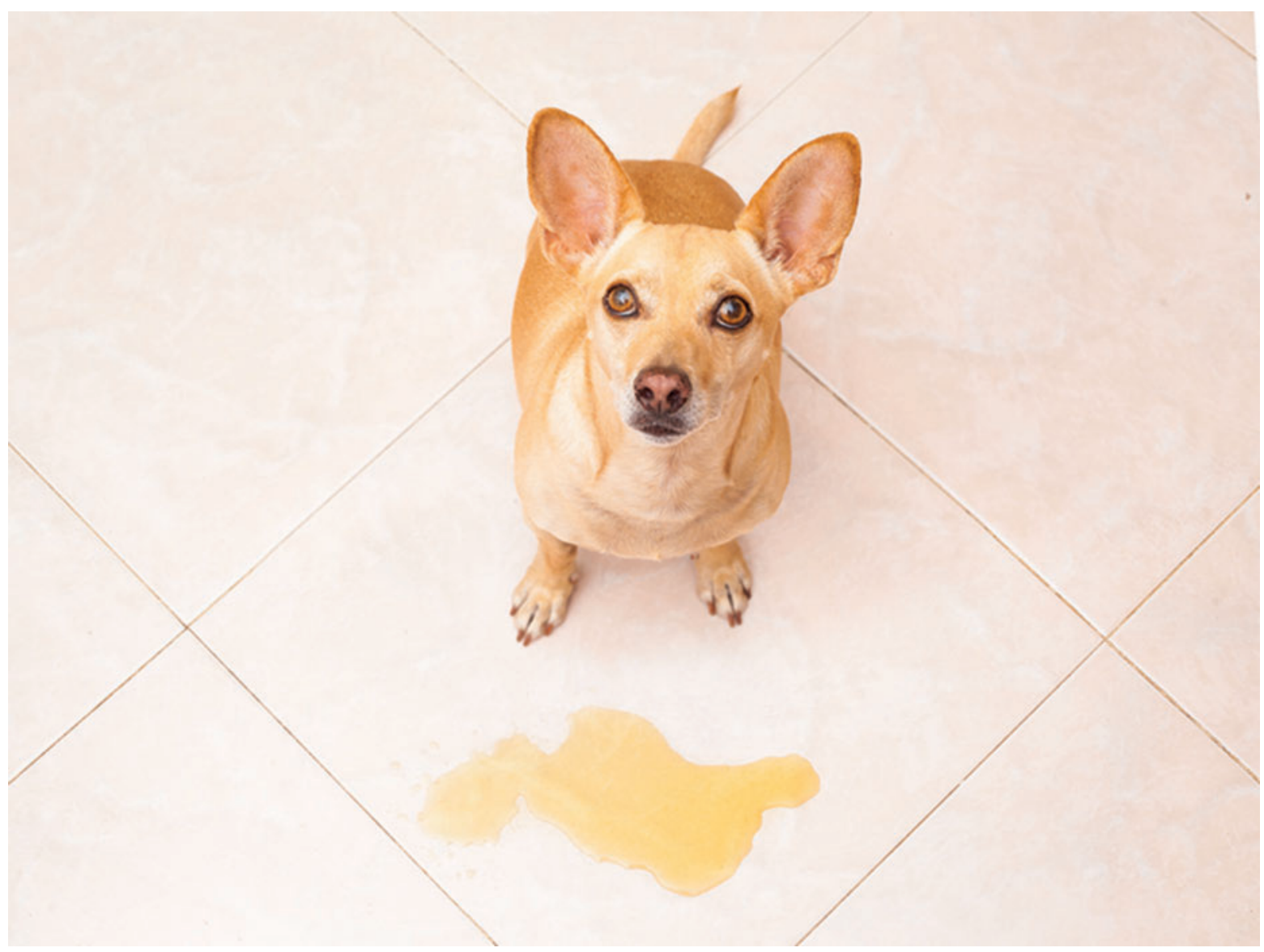

Harninkontinenz beim Hund kann viele Ursachen haben. Neben einer schulmedizinischen Versorgung bietet die Chiropraktik gute Behandlungsmöglichkeiten. Quelle: ๑ Javier brosch - stock.adobe.com [rerif] 


\section{Einleitung}

Ein in der Praxis vorgestellter Hund mit Inkontinenz sollte immer zunächst schulmedizinisch abgeklärt werden. Das absolute Minimum dabei sollten eine Allgemeinuntersuchung sowie die Untersuchung des Urins sein. Ergeben sich sowohl bei der klinischen Untersuchung als auch bei der Harnsediment- und Harnstick-Untersuchung keine Hinweise auf eine Blasenerkrankung, kann eine andere Ursache (Kasten: Ursachen) vorliegen.

\section{URSACHEN}

Harninkontinenz beim Hund ist ein Symptom, das viele Ursachen haben kann:

- Blasenentzündung

- Blasensteine

- neurologische Grunderkrankungen

- angeborene Missbildungen

- tumoröse Zubildungen

- kastrationsbedingte Inkontinenz bei der Hündin

Parallel zu einer schulmedizinischen Versorgung kann es sinnvoll sein, das Problem chiropraktisch anzugehen, da auch die Blasenfunktion des Körpers neuronaler Kontrolle unterliegt. Letztlich geht es immer um die Gesundheit des Patienten und dessen Lebensqualität. Dieses Ziel kann gerade bei Inkontinenz oftmals vollständig nur in einer Kombination aus Schulmedizin mit alternativen Methoden erreicht werden. Eine sehr gute Aufklärung des Besitzers ist vonnöten, wie auch in vielen anderen Bereichen.

\section{Physiologische Grundlagen - Pipi machen ist kein Zufall!}

Physiologisch ist das Absetzen von Harn ein hochkomplexer Vorgang. Die physiologischen Grundlagen sollen hier in aller Kürze dargestellt werden. Der in der Niere produzierte Harn erreicht durch die Harnleiter die Blase, die sich vereinfacht in 3 Schichten aufbaut: Außen ist sie von Bauchfell bzw. Bindegewebe umgeben, darauf folgt der 3-schichtige Muskelmantel, der sich in mehrere Schichten mit unterschiedlichem Verlauf gliedert. Im Bereich des Blasenhalses sind bogenförmige Muskelschleifen für den Verschluss der Harnblase wichtig. Die den Innenraum auskleidende Schleimhaut besitzt eine ausgeprägte Submukosa und ein mehrschichtiges Übergangsepithel, um der Ausdehnung des Organs im gefüllten Zustand Rechnung zu tragen.

Damit der Urin in der Blase verbleiben und gespeichert werden kann, muss sich der M. detrusor entspannen, während sich der Sphinkter der Blase in Kontraktion befindet. Bewusster Urinabsatz ist nur dann möglich, wenn das feine Zusammenspiel der unterschiedlichen Strukturen funktioniert ( $>$ Tab.1, $>$ Abb.1, $>$ Abb.2). Dies unterliegt neuronaler Kontrolle. Die Blase wird sowohl durch autonome als auch durch somatische Nerven innerviert.

Der N. hypogastricus, der aus Nervenästen aus dem Ganglion mesentericum caudalis zusammengesetzt ist, führt sympathische Fasern. Zusammen mit den Nn. pelvini, die parasympathische Fasern führen und ebenfalls in die Blasenkontrolle involviert sind, beteiligt er sich an der Bildung des Beckengeflechts, Plexus pelvinus. Der Ursprung dieser Nerven liegt beim Hund im Rückenmark auf Höhe L1-4 (N. hypogastricus) und S1-3 (Nn. pelvini). Zusätzlich zur vegetativen Kontrolle ist der $\mathrm{N}$. pudendus beteiligt, der somatische Fasern führt und aus S1-3 gespeist wird.

\section{Ziele der Chiropraktik}

Die Chiropraktik ist eine Methode zur Normalisierung, Vorbeugung und Heilung und basiert auf der Tatsache, dass das Nervensystem die zentrale Instanz des Körpers darstellt. Defizite in diesem Bereich führen zu Beeinträchtigungen der Körperfunktionen, die vom Menschen üblicherweise als Einschränkung und/oder Schmerz des Bewegungsapparats wahrgenommen werden. Aber auch alle anderen Organe werden vom Nervensystem gesteuert und können unter einer Beeinträchtigung desselben leiden.

- Tab. 1 Nervenaktivität und Blasenfunktion.

\begin{tabular}{|l|l|l|l|}
\hline Zustand der Blase & Sympathikus & Parasympathikus & $\begin{array}{l}\text { N. pudendus } \\
\text { (somatisch) }\end{array}$ \\
\hline Blasenfüllung & $\begin{array}{l}\text { Aktivierung: } \\
\text { - Halsmuskeltonus } \uparrow\end{array}$ & $\begin{array}{l}\text { Hemmung: } \\
\text { Detrusormuskel } \downarrow\end{array}$ & $\begin{array}{l}\text { Aktivierung: } \\
\text { Sphinktertonus }\end{array}$ \\
\hline $\begin{array}{l}\text { Blasenentleerung } \\
\text { (Miktion) }\end{array}$ & $\begin{array}{l}\text { Hemmung: } \\
\text { Halsmuskeltonus } \downarrow\end{array}$ & $\begin{array}{l}\text { Aktivierung: } \\
\text { - Detrusormuskel } \uparrow\end{array}$ & - \\
\hline & & - Muskelfasern am Blasenhals $\uparrow \rightarrow$ Öffnung & \\
\hline
\end{tabular}




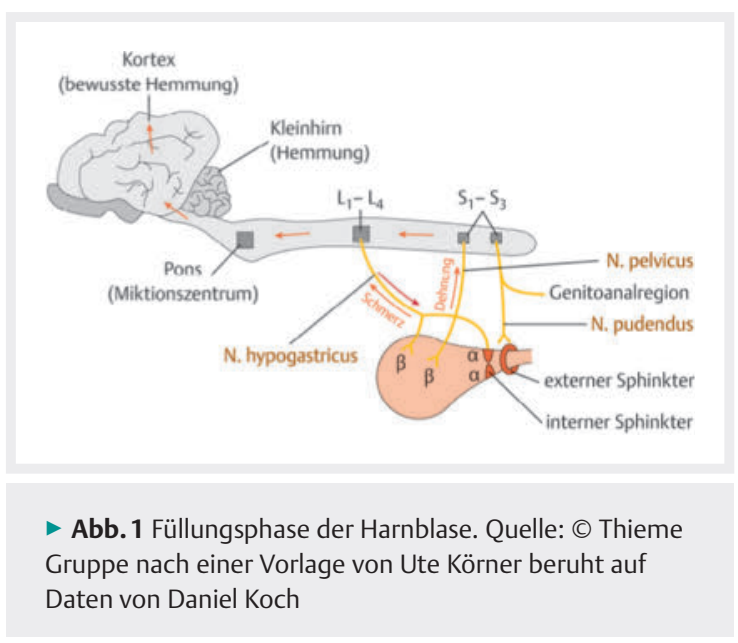

Das Ziel der chiropraktischen Behandlung ist eine optimal bewegliche Wirbelsäule, denn nur eine bewegliche Wirbelsäule lässt das Nervensystem optimal arbeiten und somit den Körper optimal funktionieren. Die chiropraktische Behandlung hat die Optimierung des Nervensystems zum Ziel. Dies wird nicht allein durch Mobilisation, v.a. der Facettengelenke erreicht. Durch die Erhöhung der Mobilität und die darauffolgende Verbesserung der Durchblutung wird auch eine bessere Nervenversorgung im Bereich des peripheren Nervensystems erreicht. Immobilisation hat eine verminderte Mobilität im Nerv selbst und eine eingeschränkte Mobilität des Nervs gegenüber dem umliegenden Gewebe zur Folge.

\section{Patient Bella}

\section{Anamnese}

Bella war eine 8-jährige weiblich-kastrierte Malinois-Hündin, die erstmals im September 2012 vorgestellt wurde. Die Hündin wurde bis Mitte des Jahres intensiv und erfolgreich im Schutzhundesport eingesetzt. Die Kastration erfolgte 2011 aufgrund einer Pyometra.

In den letzten 4-6 Wochen ist aufgefallen, dass die Hündin manchmal schlechter als früher aufsteht. Des Weiteren ist sie hin und wieder inkontinent (in Ruhe, in Form von Tröpfeln). Im Schlaf verliert sie auch mal große Mengen Urin. Es erfolgte ein Behandlungsversuch der Inkontinenz vom Haustierarzt mit Estriol, der ohne Erfolg verlief, sodass das Medikament wieder abgesetzt wurde.

Die Hündin wurde 10 Tage vor der Vorstellung in meiner Praxis geröntgt, da sich das Gangbild nach langen Spaziergängen am Strand verschlechtert hatte. Auch die Aufstehschwierigkeiten wurden deutlicher. Die Hündin sprang schlechter ins Auto. Die beeindruckenden Spondylosen ( $\triangleright$ Abb.3) hatten den Hund bis dato nicht nennenswert in seiner Bewegung eingeschränkt. Laut Aussage der Besitzerin steht die Hündin stark im Trieb

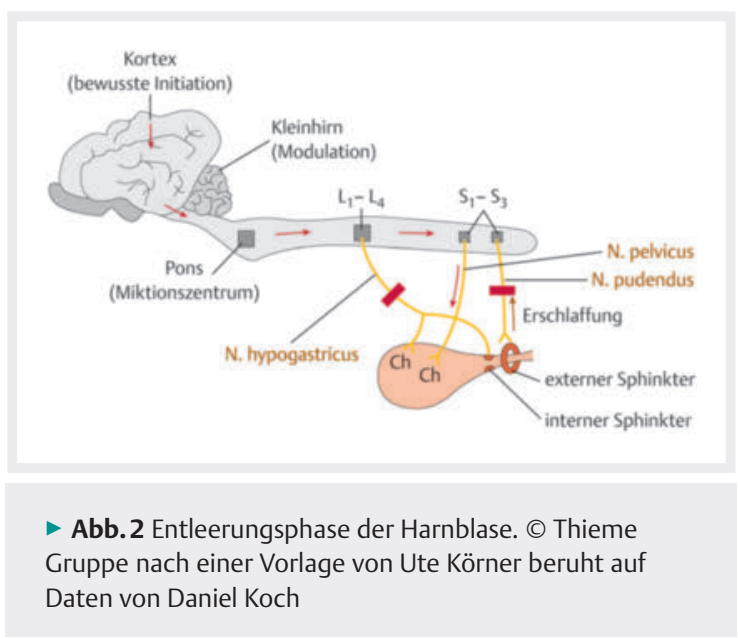

und ist auf dem Platz durch Nichts zu halten. Infolge der röntgenologischen Befunde an der Wirbelsäule wurde der Sport deutlich reduziert.

Die Hündin bekam zum Zeitpunkt der Erstvorstellung Rimadyl. Der Besitzerin zufolge hatte dies zwar positive Auswirkungen auf das Gangbild, führte jedoch auch nach 10 Tagen nicht zu einer Verbesserung der Spring- und Aufstehfähigkeiten.

\section{Untersuchung}

Erstvorstellung

\section{Befunde}

Im Stand erscheint die Rückenlinie sehr gerade, alle Gliedmaßen werden gleichmäßig belastet. Im Schritt wie im Trab werden beide Hintergliedmaßen nur wenig gebeugt. Insgesamt scheinbare Gewichtsverlagerung nach vorne. Auf gebogener Linie ist kein Unterschied auf der rechten oder linken Hand zu sehen.

Palpatorisch stark angespannt am ganzen Körper, v. a. im Bereich der Lendenwirbelsäule und des lumbosakralen

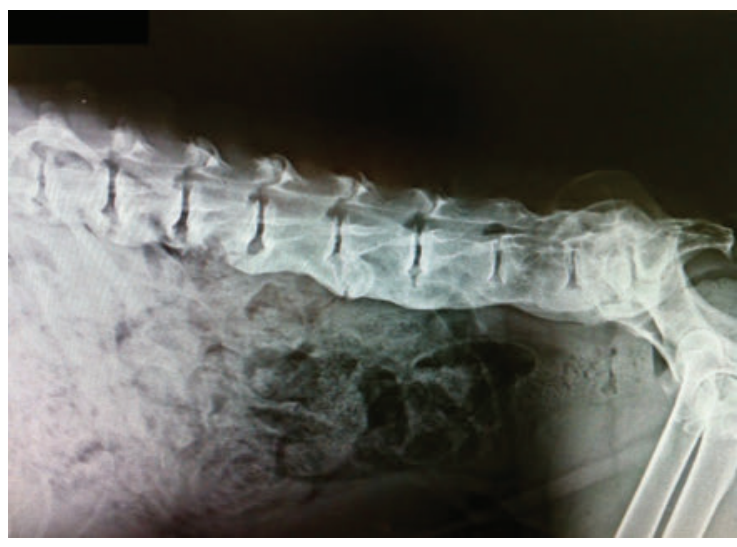

- Abb. 3 Auf dem Röntgenbild der Hündin sind deutliche Spondylosen im Bereich der Lendenwirbelsäule sichtbar. (c) Tiergesundheitszentrum Isernhagen 
Übergangs. Dabei ist schwer zu unterscheiden, ob dies schmerzbedingt oder durch eine überdurchschnittlich stark ausgeprägte Tierarztabneigung verursacht wird. Es finden sich funktionelle Fehlstellungen in der gesamten Lendenwirbelsäule, Blockaden der Iliosakralgelenke, v. a. links in Form von posterior inferior. Die Blockaden wurden chiropraktisch gelöst.

\section{Weiteres Prozedere}

Nach der Behandlung sollte die Hündin noch etwas spazieren gehen. Die Besitzer sollten in den nächsten 2 Tagen verstärkt auf das Gangbild achten, v.a. auf einen eventuell auftretenden Muskelkater.

\section{Konsultation}

Die 2. Konsultation erfolgte 17 Tage nach der Erstvorstellung. Die Hündin hatte keine negativen Nebenwirkungen von der Erstbehandlung. Sie war für 10 Tage nach der Behandlung nicht inkontinent, dann ist wieder Harnträufeln beobachtet worden. Das Gangbild erschien zu diesem Zeitpunkt wieder flüssiger. Das Rimadyl war in der Zwischenzeit ohne negative Auswirkungen ausgeschlichen worden.

\section{Befunde}

Folgende Wirbel wurden chiropraktisch manipuliert:

- L2-4 posterior (Schwingen nach oben und unten blockiert), posterior rechts (Biegung nach rechts blockiert)

- Sacral Base posterior

- Iliosakralgelenk links geringgradig posterior inferior (Blockade des Iliosakralgelenks in Beugestellung)

\section{Weiteres Prozedere}

Hausaufgaben für die Besitzerin:

- tägliche Wärmepackungen für den Rücken und die Kruppe

- tägliche Rückenmassage

\section{Merke}

Die Hausaufgaben sollten bei allen Patienten nur so lange gemacht und so oft wiederholt werden, wie der Hund es gut toleriert.

\section{Konsultation}

Die 3. Konsultation erfolgte 4 Monate nach der Erstvorstellung. Die Hündin war bis vor wenigen Tagen kontinent, seither bestanden auch wieder Schwierigkeiten beim Aufstehen. Gangbild deutlich verschlechtert, daher erfolgte auch vor dem Hintergrund der röntgenologischen Befunde der Rat zur parallelen Therapie mit NSAID durch den Haustierarzt.

\section{Befunde}

Folgende Wirbel wurden chiropraktisch manipuliert:

- L1-3 posterior, posterior rechts

- C4, C5 Body left

\section{Weiteres Prozedere}

Hausaufgaben für die Besitzerin:

- Wärmepackungen für den Rücken und die Kruppe

- Rückenmassage

- schonendes Durchbewegen, v. a. der Hintergliedmaßen in entspannter Seitenlage (war in der Praxis nicht möglich)

Die Hausaufgaben sind täglich oder zumindest mehrmals wöchentlich durchzuführen, sofern sie gut toleriert werden.

\section{Folgetermine}

In der Folge wurde die Hündin immer dann vorgestellt, wenn wahlweise die Inkontinenz mehr wurde oder eine Bewegungseinschränkung in Form von schwererem Aufstehen oder auch steiferem Gang auffielen. Das Rimadyl wurde noch einmal abgesetzt, jedoch schien eine Dauermedikation sinnvoll. Die Inkontinenz war zwar insgesamt deutlich besser (weniger in Menge und Inzidenz), verschlechterte sich aber schleichend. Eine chirurgische Intervention in Form einer Kollageninjektion oder Kolposuspension kam für die Besitzerin nicht infrage.

\section{AUF DEN PUNKT GEBRACHT}

Das Besondere an diesem Fall ist meiner Ansicht nach die beeindruckende Spondylosenbildung, die lange Zeit ohne nennenswerte Einschränkung der Lebensqualität der Hündin verlief. Dies hängt offensichtlich mit dem Wesen des jeweiligen Hundes zusammen. Diese Hündin war ein echtes Arbeitstier. Des Weiteren beachtenswert ist, dass sich trotz der starken Veränderungen der Wirbelsäule selbige auch noch in diesem Bereich chiropraktisch beeinflussen ließ.

Außerdem ist der Fall ein gutes Beispiel dafür, dass die Zusammenarbeit von Haustierarzt und Chiropraktiker vonnöten ist, um das Optimum für den Patienten zu erreichen. Die Chiropraktik allein hat zu einem fortgeschrittenen Zeitpunkt nicht mehr ausgereicht, um das Optimum an Lebensqualität für die Hündin zu erreichen. Genauso reichten zu Beginn der Therapie die NSAID nicht aus, um einen schmerzfreien Zustand der Hündin zu erreichen.

Ziel der Behandlung eines Hundes mit derartigen Befunden sollte immer die lange Erhaltung der Lebensqualität sein. Dies ist durch eine schulmedizinische Behandlung in Verbindung mit chiropraktischen, osteopathischen und physiotherapeutischen Maßnahmen möglich sowie durch den begleitenden Einsatz von Medikamenten zur Schmerzreduktion und zur begleitenden Behandlung der Inkontinenz. 


\section{Patient Bello}

\section{Anamnese}

Bello war ein 9-jähriger männlich-kastrierter BorderSpitz-Mischling ( Abb.4), der erstmalig im Mai 2012 vorgestellt wurde. Der Hund wurde 2004 kastriert, ist regelmäßig entwurmt und geimpft und hat keinerlei bekannte Erkrankungen. Die Besitzerin spielt mit dem Hund Frisbee. Er zeigt sich aber zunehmend unmotiviert. Das gravierendste Symptom, das auch zur Vorstellung des Hundes in meiner Praxis geführt hat, ist jedoch die zunehmende Aggressivität gegenüber Artgenossen. Erst im Gespräch wird auch die Inkontinenz erwähnt, die Bello seit der Kastration vor 8 Jahren (!) im Schlaf zeigt.

\section{Untersuchung}

\section{Erstvorstellung}

\section{Befunde}

Im Stand erscheint die Rückenlinie unauffällig, alle Gliedmaßen werden gleichmäßig belastet. Im Schritt wie im Trab wird die rechte Hintergliedmaße geringgradig weniger gebeugt im Vergleich zur linken Hintergliedmaße, ohne jedoch eine Lahmheit im schulmedizinischen Sinne zu zeigen. Auf gebogener Linie zeigt sich kein Unterschied zwischen der rechten und der linken Hand.

Das Fell in der Ellenbogenbeuge ist beidseits rötlich verfärbt, laut Besitzerin ist dies „schon immer“ der Fall. Die Haut erscheint in diesem Bereich ohne besonderen Befund, die Stellen werden laut Besitzerin nicht vermehrt geleckt.

Untersuchung und Behandlung von Bello sind nur mit Maulkorb möglich, mit Maulkorb ist der Hund aber umgänglich. Palpatorisch sind die Muskulatur der Lendenwirbelsäule sowie die Rückenfaszie sehr fest. Die Haut im Bereich der Lendenwirbelsäule lässt sich kaum verschieben. Die Beweglichkeit aller Gliedmaßen ist symmetrisch unauffällig.

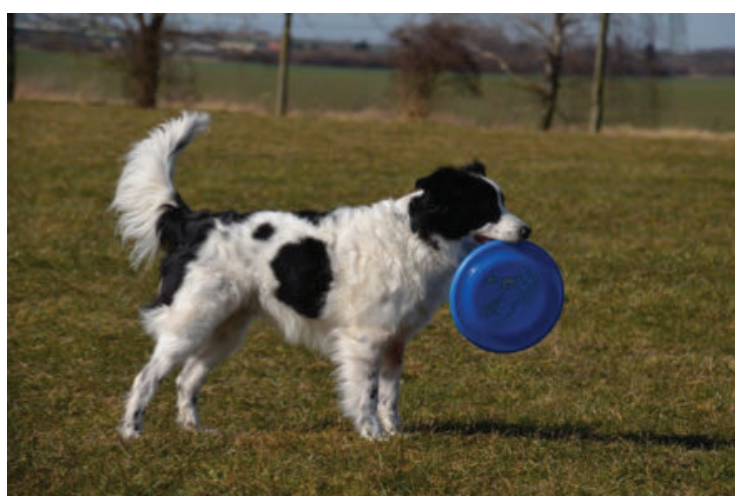

- Abb. 4 Bello zeigte sich zunehmend unmotiviert-auch beim Spiel mit dem Frisbee. Quelle: ๑ Antje Beinhoff
Folgende Wirbel wurden chiropraktisch manipuliert:

- L1-3 posterior

- Blockade der Iliosakralgelenke, v. a. rechts in Form von posterior inferior

\section{Weiteres Prozedere}

Hautverschiebungen im Bereich der Lendenwirbelsäule gezeigt. Durch Auflegen der Hand im Bereich der Lendenwirbelsäule und flächenhafte Verschiebung der Haut auf dem Rücken wird einerseits ein Wärmeeffekt erzielt, andererseits hat man so über die gedehnten Fasern der Unterhaut auch einen Effekt auf die Faszien im behandelten Bereich. Die Besitzerin soll die Übungen täglich (gerne auch mehrfach) zuhause machen, sofern Bello es gut toleriert.

\section{Konsultation}

Die 2. Konsultation erfolgte erst 6 Monate nach Erstvorstellung, da die Anreise für die Besitzerin sehr lang war. In der Zwischenzeit wurde der Hund unregelmäßig physiotherapeutisch betreut. Bello hatte nach der Erstbehandlung keine Nebenwirkungen in Form von Muskelkater o.ä. gezeigt. Das Verhalten gegenüber Artgenossen hatte sich verbessert. Die Inkontinenz bestand nach wie vor. Sie war auf Nachfrage bei der Besitzerin nach dem Erstbesuch zunächst geringer, dies ließ sich aber nicht genau beziffern.

\section{Befunde}

Folgende Wirbel wurden chiropraktisch manipuliert:

- T10-L2 posterior

- L2 und L3 posterior rechts

- Iliosakralgelenke beiderseits geringgradig posterior inferior

\section{Weiteres Prozedere}

Hausaufgaben wie gehabt.

\section{Konsultation}

Die 3. Konsultation erfolgte 10 Monate nach der letzten Behandlung. Vor 14 Tagen zeigte der Hund eine Lahmheit vorne links für 1-2 Tage. Bello ging ohne Behandlung (Physiotherapie oder Schulmedizin) wieder lahmfrei, war in letzter Zeit aber wieder vermehrt inkontinent. Bis 3-4 Wochen vor der 3. Konsultation war Bello komplett kontinent.

\section{Befunde}

Folgende Wirbel wurden chiropraktisch manipuliert:

- Iliosakralgelenk posterior inferior links

- L2-4 posterior

- L3 posterior links

- T11 posterior rechts

\section{Weiteres Prozedere}

Hausaufgaben wie gehabt. Ab diesem Zeitpunkt nach intensiver Beratung regelmäßige Behandlung alle 3-5 Monate mit dem Erfolg, dass die Inkontinenz bis auf 
Ausnahmefälle, z.B. nach erhöhter Belastung/Überlastung, nicht mehr auftrat. Parallel zur Chiropraktik wurde der Hund ab 2015 immer mal wieder einer Physiotherapeutin vorgestellt. Die Behandlungen dort erfolgten 3mal innerhalb von 3 Wochen, dann wurden wieder Pausen gemacht.

\section{AUF DEN PUNKT GEBRACHT}

Der Fall war insofern ungewöhnlich, als dass Inkontinenz bei Rüden zumindest in meiner Praxis nicht so häufig vorkommt. Außerdem ist dieser Fall ein eindrucksvolles Beispiel dafür, dass Kommunikation und in der Folge (hoffentlich) eine gute Compliance der Besitzer unumgänglich sind. Die Besitzerin hatte nicht auf Verbesserung der Inkontinenz gehofft, dies war nicht Bestandteil der Gründe für die Erstvorstellung. Sie hat die Verbesserung nach mehreren Behandlungen überhaupt erst mit der Chiropraktik in Verbindung gebracht und war dann auch bereit, Bello öfter vorzustellen.

\section{Patient Elsa}

\section{Anamnese}

Elsa ist eine Greyhoundhündin ( $\triangleright$ Abb.5), die im August 2016 geboren und erstmalig im Mai 2019 vorgestellt wurde. Zu diesem Zeitpunkt war die Hündin unkastriert. Elsa wurde im letzten Jahr vereinzelt auf der Rennbahn eingesetzt, hatte schon mehrere Kapselverletzungen an den Zehengelenken, manchmal bestand der Verdacht auf Rückenschmerzen. In den letzten 4 Wochen ist es 2mal nach der morgendlichen Gassirunde und

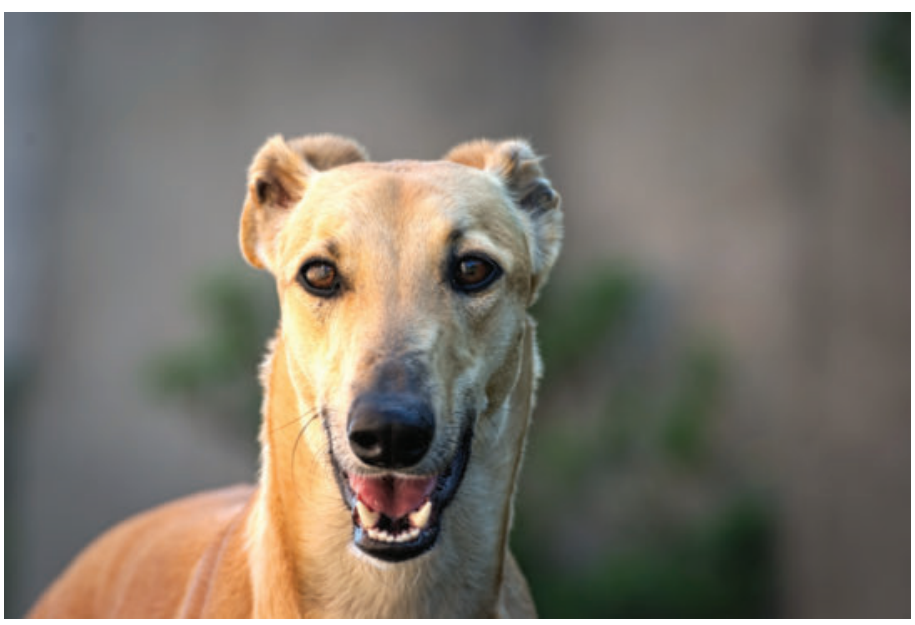

- Abb.5 Elsa ist eine Greyhoundhündin, die auch auf der Rennbahn eingesetzt wurde. (Symbolbild). Quelle: @ [rerif] anschließendem Fressen zu Urinverlust gekommen. Die Hündin ist schon früher aufgrund von Blasenentzündungen in Behandlung gewesen, im Dezember 2017 wurde Blasengrieß festgestellt. Danach wurde sie mit entsprechendem Diätfutter für ca. 4 Monate ernährt. Jetzt wieder Fütterung mit normalem Futter, um dem Energiebedarf eines Sporthunds gerecht zu werden.

\section{Untersuchung}

\section{Erstvorstellung}

Physiologische Körperhaltung und Belastung aller Gliedmaßen sowohl im Stand als auch in der Bewegung. Hochgradig Zahnstein und v.a. im vorderen Bereich mittel- bis hochgradige Zahnfleischentzündung. Daher Rat zur Zahnsanierung durch den Haustierarzt. Wiedervorstellung nach der Sanierung unter Narkose angeraten.

\section{Befunde}

Folgende Wirbel wurden chiropraktisch manipuliert:

- L1-5 posterior

\section{Weiteres Prozedere}

Tägliche Hautverschiebungen im Bereich der Lendenwirbelsäule gezeigt. Diese soll die Besitzerin zuhause durchführen, sofern Elsa es gut toleriert.

\section{Konsultation}

Die 2. Konsultation fand 6 Wochen nach der Erstvorstellung statt. Urinverlust war nicht mehr aufgetreten. Vor 3 Wochen Zahnsanierung und Entfernung der Eierstöcke. Die Naht ist komplikationslos geheilt, seit 1 Woche registriert die Besitzerin aber wieder geringgradigen Maulgeruch.

\section{Befunde}

Folgende Wirbel wurden chiropraktisch manipuliert:

- L2-4 posterior

- Iliosakralgelenk links posterior inferior

\section{Weiteres Prozedere}

Hautverschiebungen und Massage der Muskulatur der Lendenwirbelsäule, v.a. nach Trainingseinheiten angeraten.

\section{Konsultation}

Der 3. Besuch fand 14 Tage nach der letzten Behandlung statt. Die Hündin zeigte keinen Urinverlust, es fiel aber eine feuchte Scheide auf, sodass die Besitzerin eine Wiedervorstellung der Hündin wünschte, bevor sie wieder massiv inkontinent werden würde.

\section{Befunde}

Folgende Wirbel wurden chiropraktisch manipuliert:

- L3 posterior

- Iliosakralgelenk links geringgradig posterior inferior 


\section{Weiteres Prozedere}

Hautverschiebungen und Massage der Muskulatur der Lendenwirbelsäule, v.a. nach Trainingseinheiten angeraten.

\section{Konsultation}

Die 4. Kontrolle fand 3 Wochen nach der letzten Behandlung statt. Laut Besitzerin war nach dem 1. und 2. Trainingslauf noch alles gut. Nach 3. Trainingslauf auf schwerem Boden hechelte die Hündin lange Zeit und die Hinterbeine wirkten kraftlos. Zuhause war Elsa einmalig wieder inkontinent. Die Behandlung erfolgt 3 Tage nach dem Vorfall, zu diesem Zeitpunkt hatte sich die Hündin erholt, wirkte nicht mehr kraftlos und war nicht wieder inkontinent.

\section{Befunde}

Folgende Wirbel wurden chiropraktisch manipuliert:

$$
\text { - L2-3 posterior }
$$

\section{Weiteres Prozedere}

Erneute Überweisung an den Haustierarzt zur Kontrolle des Blasenstatus. Harnstick und Sediment auf Nachfrage ohne besonderen Befund. Die Inkontinenz ist nicht wieder aufgetreten, dennoch soll demnächst eine Ultraschalluntersuchung zur weiteren Abklärung erfolgen. Zuletzt zeigte sich die Hündin kontinent und leistungsstark.

\section{AUF DEN PUNKT GEBRACHT}

Dies ist ein spannender Fall, da die Inkontinenz zwar vergleichsweise früh auftrat, eine angeborene Anomalie jedoch weitestgehend ausgeschlossen werden kann, da man die Probleme dann noch früher erwartet hätte. Zudem war die Hündin zum Zeitpunkt des ersten Auftretens der Inkontinenz noch nicht kastriert, sodass auch diese Ursache ausgeschlossen werden konnte.

Die Tatsache, dass in allen $\mathbf{3}$ geschilderten Fällen Rückfälle häufig in Zusammenhang mit starker Belastung respektive Überlastung auftraten, könnte vermuten lassen, dass unter anderem eine starke Überdehnung des Grenzstrangs und / oder der peripheren Nerven im Bereich der Brust- und Lendenwirbelsäule eine vorübergehende Überreizung der beteiligten Nerven nach sich zieht. Die Inkontinenz wird vom Besitzer häufig nicht mit Problemen des Nervensystems und/oder der Wirbelsäule in Verbindung gebracht. Zeigt der Hund keinerlei Einschränkungen in der Bewegung, denken die Besitzer häufig, dass Wirbelsäule und Nervensystem in Ordnung sein müssten.

\section{Fazit}

Die Inkontinenz des Hundes kann vielfältige Ursachen haben, sodass zunächst immer eine schulmedizinische Abklärung empfehlenswert ist. Um eine gute Lebensqualität des Hundes zu ermöglichen und den Leidensdruck für Besitzer und Hund zu senken, ist therapeutisch eine Kombination aus Schulmedizin und alternativen Methoden empfehlenswert. Eine gute Aufklärung des Besitzers ist ratsam, um eine optimale Compliance und einen guten Therapieerfolg zu erreichen.

Autor

\section{Dr. Ute Körner}

Fachtierärztin für Chiropraktik (A); 2000 Promotion Tierärztliche Hochschule Hannover; 2001-2005 angestellt in verschiedenen Praxen für Pferde und Kleintiere; 2004

Zusatzausbildung Chiropraktik; seit 2005 in eigener Praxis ausschließlich chiropraktisch tätig; info@utekoerner.de

\section{Literatur}

[1] Bockstahler B, Levine D, Millis D. Physiotherapie auf den Punkt gebracht. Babenhausen: VBS VetVerlag; 2004

[2] Koch D. Grundlagen der Neurologie der Blase. Im Internet: https://dkoch.ch/fileadmin/user_upload/Neurologische\% 20Probleme/Neurologie_Blase_Operationen_Koch.pdf; Stand: Oktober 2019

[3] McGovern C, Goff L. Animal Physiotherapy. Chichester: WileyBlackwell; 2016

[4] Nickel R, Schummer A, Seiferle E. Lehrbuch der Anatomie der Haustiere, Band IV: Nervensystem, Sinnesorgane, Endokrine Drüsen. 4. Aufl. Stuttgart: Parey; 2003

[5] Nickel R, Schummer A, Seiferle E. Lehrbuch der Anatomie der Haustiere, Band II: Eingeweide. 9. Aufl. Stuttgart: Parey; 2004

\section{Bibliografie}

DOI https://doi.org/10.1055/a-1009-6301

Hands on 2019; 1: 39-45

(c) Georg Thieme Verlag KG Stuttgart · New York

ISSN 2628-6033 